\title{
Acid-Sensing lon Channel 1a Regulates Fate of Rat Nucleus Pulposus Cells in Acid Stimulus Through Endoplasmic Reticulum Stress
}

\author{
Zhi-Yang Xie, ${ }^{1, \dagger}$ Lu Chen, ${ }^{1, \dagger}$ Cong Zhang, ${ }^{1, \dagger}$ Lei Liu, ${ }^{1}$ Feng Wang, ${ }^{1}$ Feng Cai, ${ }^{1,2}$ Xiao-Hu Wang, ${ }^{1}$ Rui Shi, \\ Arjun Sinkemani, ${ }^{1}$ Hao-Min Yu, ${ }^{1}$ Xin Hong, ${ }^{1, *}$ and Xiao-Tao $\mathrm{Wu}^{1, *}$
}

\begin{abstract}
Acid-sensing ion channel 1a (ASIC1a) participates in human intervertebral disc degeneration (IVDD) and regulates the destiny of nucleus pulposus cells (NPCs) in acid stimulus. However, the mechanism of ASIC1a activation and its downstream pathway remain unclear. Endoplasmic reticulum (ER) stress also participates in the acidinduced apoptosis of NPCs. The main purpose of this study was to investigate whether there is any connection between ASIC1a and ER stress in an acid-induced nucleus pulposus degeneration model. The IVDs of SpragueDawley rats were stained by immunohistochemical staining to evaluate the expression of ASIC1a in normal and degenerated rat nucleus pulposus. ASIC1a expression was also quantified by quantitative real-time-polymerase chain reaction and Western blotting analysis. NPCs were exposed to the culture media with acidity at pH 7.2 and 6.5 for $24 \mathrm{~h}$, with or without 4-phenylbutyrate (4-PBA, a blocker of the ER stress pathway). Cell apoptosis was examined by Annexin V/Propidium lodide (PI) staining and was quantified using flow cytometry analysis. ASIC1amediated intracellular calcium was determined by $\mathrm{Ca}^{2+}$ imaging using Fura-2-AM. Acidity-induced changes in ER stress markers were studied using Western blotting analysis. In vivo, ASIC1a expression was upregulated in natural degeneration. In vitro, acid stimulus increased intracellular calcium levels, but this effect was blocked by knockdown of ASIC1a, and this reversal was partly inhibited by 4-PBA. In addition, blockade of ASIC1a reduced expression of ER stress markers, especially the proapoptotic markers. ASIC1a partly regulates ER stress and promotes apoptosis of NPCs under acid stimulus and may be a novel therapeutic target in IVDD.
\end{abstract}

Keywords: acid-sensing ion channel 1a; apoptosis; endoplasmic reticulum stress; intervertebral disc degeneration; nucleus pulposus

\section{Background}

Low back pain is strongly associated with intervertebral disc degeneration (IVDD) and imposes significant social and economic burdens. ${ }^{1-3}$

Within the anaerobic environment of the intervertebral disc (IVD), nucleus pulposus cells (NPCs) undertake glycolysis depending on their endoplasmic reticulum (ER) function, and the IVD is thus characterized by high concentrations of lactic acid and low $\mathrm{pH} .{ }^{4}$ Lactic acid, in turn, triggers ER stress by disrupting protein folding and calcium homeostasis. ${ }^{5-7}$ Its protein maturation machinery and other energy-intensive processes make the ER highly sensitive to numerous factors, including toxins, nutrition, and energy deprivation, redox imbalances, and disorder in $\mathrm{Ca}^{2+}$ storage. ${ }^{8}$

As members of the degenerin/epithelial $\mathrm{Na}^{+}$channel superfamily, acid-sensing ion channels (ASICs) are $\mathrm{H}^{+}$ gated voltage insensitive. To date, six ASIC subunits

\footnotetext{
${ }^{1}$ Department of Spine Surgery, ZhongDa Hospital, School of Medicine, Southeast University, Nanjing, China.

${ }^{2}$ Department of Orthopedics, First Affiliated Hospital of Soochow University, Suzhou, China.

†The first three authors contributed equally.

*Address correspondence to: Xiao-Tao Wu, MD, Department of Spine Surgery, Zhongda Hospital, School of Medicine, Southeast University, No. 87, Dingjiaqiao Road, Nanjing 210009, China, E-mail: wuxiaotao@medmail.com.cn or Xin Hong, MD, Department of Spine Surgery, Zhongda Hospital, School of Medicine, Southeast University, No. 87, Dingjiaqiao Road, Nanjing 210009, China, E-mail: xinhong102400@163.com
} 
have been identified-ASIC1a, ASIC1b, ASIC2a, ASIC2b, ASIC3, and ASIC4-and are encoded by four ASIC genes. ${ }^{9}$ Homomeric ASICla is $\mathrm{Ca}^{2+}$ and $\mathrm{Na}^{+}$permeable, whereas other subunit combinations are only $\mathrm{Na}^{+}$permeable. The $\mathrm{pH}$ values required for half-maximal activation of ASICs $\left(\mathrm{pH}_{0.5}\right)$ vary from 4.9 to $6.8 .^{10}$

According to results of our previous study, ${ }^{5}$ activation of $\mathrm{Ca}^{2+}$-permeable ASIC1a participates in IVDD and regulates the destiny of human NPCs in acid stimulus. We have also demonstrated that ER stress participates in the survival of cells during acid-induced NPCs apoptosis. ${ }^{6}$ However, it remains unclear if ASICla directly mediates $\mathrm{Ca}^{2+}$ influx and increased intracellular $\mathrm{Ca}^{2+}$ $\left[\mathrm{Ca}^{2+}\right]_{\mathrm{i}}$ in rat NPCs. Direct evidence for a causal role of ASICla-mediated $\mathrm{Ca}^{2+}$ entry in ER stress is lacking.

In the present study, we tested the hypothesis that extracellular acidosis increases NPCs apoptosis through ASICla-mediated calcium entry and the subsequent activation of ER stress. The main purpose of this study was to determine the relationship between ASICla and ER stress in NPCs under acid stimulus.

\section{Materials and Methods}

All procedures specified below were approved by the Institutional Animal Care and Use Committee of Southeast University (Nanjing, China, 2016ZDKYSB089).

\section{In vivo studies}

Sprague-Dawley rats (24 males, age 8-40 weeks) were euthanized with $\mathrm{CO}_{2}$ and were examined by magnetic resonance imaging (MRI, 7T; Bruker, Germany) to evaluate the level of degeneration. Following imaging, the spine was collected from midthoracic to lumbar segments. After fixation, decalcification, and embedding in paraffin, coronal sections $3 \mu \mathrm{m}$ in thickness were prepared according to methods described in our previous study. ${ }^{11}$ The sections were stained with the conventional Hematoxylin and Eosin (H\&E) staining method.

\section{Immunohistochemical and}

immunofluorescence staining

Deparaffinized coronal sections were blocked with 5\% bovine serum albumin (BSA) in TBST (10 mM Tris, $\mathrm{pH}$ 8.0, $150 \mathrm{mM} \mathrm{NaCl}$, and $0.1 \%$ Tween 20 ) and were incubated with primary antibodies at $4{ }^{\circ} \mathrm{C}$ overnight. The sections were subsequently incubated with secondary antibody diluted in phosphate-buffered saline (PBS) (A0208, 1:5000, Beyotime, China; Alexa Fluor 594, 1:1000, Invitrogen) for $1.5 \mathrm{~h}$ at room temperature. Immunohistochemical stain- ing was viewed with light microscopy (Olympus BX41, Japan). For immunofluorescence staining, 4,6-diamidino2-phenylindole (DAPI; Sigma-Aldrich, Ireland) was used to counterstain cell nuclei for $5 \mathrm{~min}$ at room temperature, and the cells were observed with a fluorescence microscope (Olympus, Japan). For the negative control, the same procedure was performed, omitting incubation with primary antibodies.

\section{In vitro studies}

Isolation and culture of NPCs. Sprague-Dawley rats (20 males, age 8-40 weeks) were euthanized with $\mathrm{CO}_{2}$. NP tissues were collected as previously described. ${ }^{11,12}$ The incompletely digested NP was maintained in DMEM/ F-12 (GIBCO, Grand Island, NYA) containing 10\% FBS (Elite Biotech, Germany) and 1\% penicillin/streptomycin (GIBCO) and cultured in $95 \%$ humidity, $21 \% \mathrm{O}_{2}$, and $5 \% \mathrm{CO}_{2}$ at $37^{\circ} \mathrm{C}$. Confluent cells were lifted using $0.25 \%$ Trypsin-EDTA $(1 \times)$ solution (Life Technologies, Canada) and were then subcultured (1:2) to collect passage 1 (P1) and P2 cells. The P2 NPCs were used in the subsequent assays.

RNA interference of ASIC1a. We used retroviral vectors containing ASICla-siRNA (constructed by Hanbio, China), and the retrovirus-expressing blank served as a negative control. A monolayer of NPCs with $70 \%$ confluence were infected with the retroviruscontaining supernatant in the presence of polybrene $(6 \mu \mathrm{g} / \mathrm{mL}$, Hanbio) for $24 \mathrm{~h}$, and then were exposed to puromycin $(2 \mu \mathrm{g} / \mathrm{mL}$, Hanbio). Western blot analysis was performed to evaluate levels of RNA interference. At 5 days after infection, cells were subjected to acid treatment.

Exposure of NPCs to acid. As described in our previous studies, ${ }^{5,6} \mathrm{pH} 6.5$ was selected as a mimic of degenerated IVD, and $\mathrm{pH} 7.2$ was used as the control. Cells were incubated with 4-phenylbutyrate (4-PBA) ( $5 \mathrm{mM}$; Sigma) for $24 \mathrm{~h}$ before acid treatment. The cells were then harvested after exposure to the acidic condition.

Detection of activation of ASIC1a by Fura-2-AM. As described in our previous studies, cells on glass bottom dishes (Cellvis) were washed with PBS twice and then incubated with Fura-2-AM ( $5 \mu \mathrm{M}$; Sigma, Ireland) for $30 \mathrm{~min}$ at room temperature. After washing twice again, Fura-2-AM fluorescence was observed with a laser scanning confocal microscope (Olympus) with excitation at $488 \mathrm{~nm}$ and emission at $525 \mathrm{~nm}$. Observation 
continued when the acidic solution ( $\mathrm{pH}$ 6.5) was added and continued for $10 \mathrm{~min}$ thereafter. The mean fluorescence value was calculated and the intensity of the fluorescence was measured by LSM 5 Image software.

Apoptotic analyses by flow cytometry. Apoptosis analyses were performed using the Annexin V-Fluorescein Isothiocyanate (FITC)/Propidium Iodide (PI) DualStaining Kit according to the manufacturer's instructions (KeyGen, China). Floating and attached cells were collected, resuspended in $500 \mu \mathrm{L}$ binding buffer, and incubated with $5 \mu \mathrm{L}$ Annexin V-FITC and $5 \mu \mathrm{L}$ PI for $10 \mathrm{~min}$ at room temperature in the dark. Cells were quantified by flow cytometry (BD Accuri C6).

Quantitative real-time polymerase chain reaction. NPCs were collected, and total mRNA was extracted using the Universal RNA Extraction Kit (9767; TaKaRa, Dalian, China). Reverse transcription was performed by following the manufacturer's instructions for the PrimeScript RT Reagent Kit with gDNA Eraser (RR047; TaKaRa), and real-time polymerase chain reaction (RT-PCR) was performed using SYBR Green PCR Master Mix (Roche Applied Science) in a StepOnePlus PCR System (Applied Biosystems). Gene-specific primer sequences are listed in Table 1.

Protein extraction and Western blotting. As in our previous research, ${ }^{11,13}$ total proteins were extracted using whole-cell and tissue lysis assay (KeyGen). Protein quantification using a BCA assay (Beyotime) was performed according to the manufacturer's instructions. Total proteins were resolved on $4-20 \%$ Bis-Tris gels (GenScript, China) and transferred by electroblotting onto nitrocellulose membranes (Pall). The membranes were blocked with $5 \%$ nonfat dry milk in TBST for $1.5 \mathrm{~h}$ and incubated overnight at $4{ }^{\circ} \mathrm{C}$ with primary antibodies (Table 2). After washing with TBST, the membranes were incubated for $1 \mathrm{~h}$ with secondary antibodies (ab6721, 1:5000; Abcam) diluted in $5 \%$ BSA/TBST at room temperature. Super Signal

Table 1. Gene-Specific Primer Sequences

\begin{tabular}{ll}
\hline Gene & \multicolumn{1}{c}{ Primers } \\
\hline ASIC1a & \\
Forward & CACAGATGGCTGATGAAAAGCAG \\
Reverse & CATGGTAACAGCATTGCAGGTC \\
$\beta$-actin & CCCATCTATGA GGGTTACGC \\
Forward & TाTAATGTACGCACGATTC \\
Reverse & \\
\hline
\end{tabular}

Table 2. Antibodies Used in Western Blotting and Immunohistochemistry

\begin{tabular}{ll}
\hline Target & Antibody (Company, LotID, usage) \\
\hline ASIC1a & Alomone Labs, ASC-014 (1:200) \\
GRP78 (BIP) & Abcam, ab21685 (1:1000) \\
XBP1 & Abcam, ab37152 (1:500) \\
CHOP (DDIT3) & Abcam, ab10444 (1:250) \\
Caspase12 & Abcam, ab62484 (1:500) \\
$p$-eif2 $\alpha$ & Abcam, ab32157 (1:500) \\
$t$-eif2 $\alpha$ & Abcam, ab131495 (1:500) \\
Vinculin & Abcam, ab129002(1:10000) \\
\hline
\end{tabular}

West FEMO Chemiluminescent Substrate (Thermo Scientific) was used for detection. The protein expression level was determined by densitometric analysis and normalized to the level of vinculin.

\section{Statistical analysis}

Statistical analysis was performed using SPSS 20.0. All data are expressed as the mean $\pm S D$ and were taken from multiple independent experiments. The results were compared among different groups by using oneway ANOVA and the unpaired $t$-test. $p$-Values $<0.05$ were considered as significant.

\section{Results}

Morphology of rat nucleus pulposus

$H \& E$ staining and MRI were utilized to evaluate the basic morphology of rat intervertebral discs. During the degenerative process, the number of NPCs decreased as the moisture content decreased (Fig. 1A, C).

\section{Expression of ASIC1a in rat NP}

ASICla expression in rats was determined using immunofluorescence and immunohistochemical analyses (Fig. 1B). ASICla expression was measured in healthy and degenerated IVDs by Western blot analysis and quantitative RT-PCR (Fig. 1D). In comparison with the healthy NP, expression of ASICla was significantly higher in the setting of natural degeneration, both in tissue and at cellular levels $(p<0.01, n=10)$.

\section{Activation of ASIC1a in rat NPCs under acid stimulus}

ASIC1a-siRNA was used to knock down ASIC1a of rat NPCs, and the level of interference was quantified by Western blot analysis (Fig. 2A).

To evaluate acid-induced activation of ASICla of NPCs, the $\left[\mathrm{Ca}^{2+}\right]_{\mathrm{i}}$ in NPCs was examined by Fura-2AM fluorescent $\mathrm{Ca}^{2+}$ imaging after treatment with extracellular acid. $\mathrm{Ca}^{2+}$ imaging revealed a significant increase in intracellular $\mathrm{Ca}^{2+}$ in response to an acid 


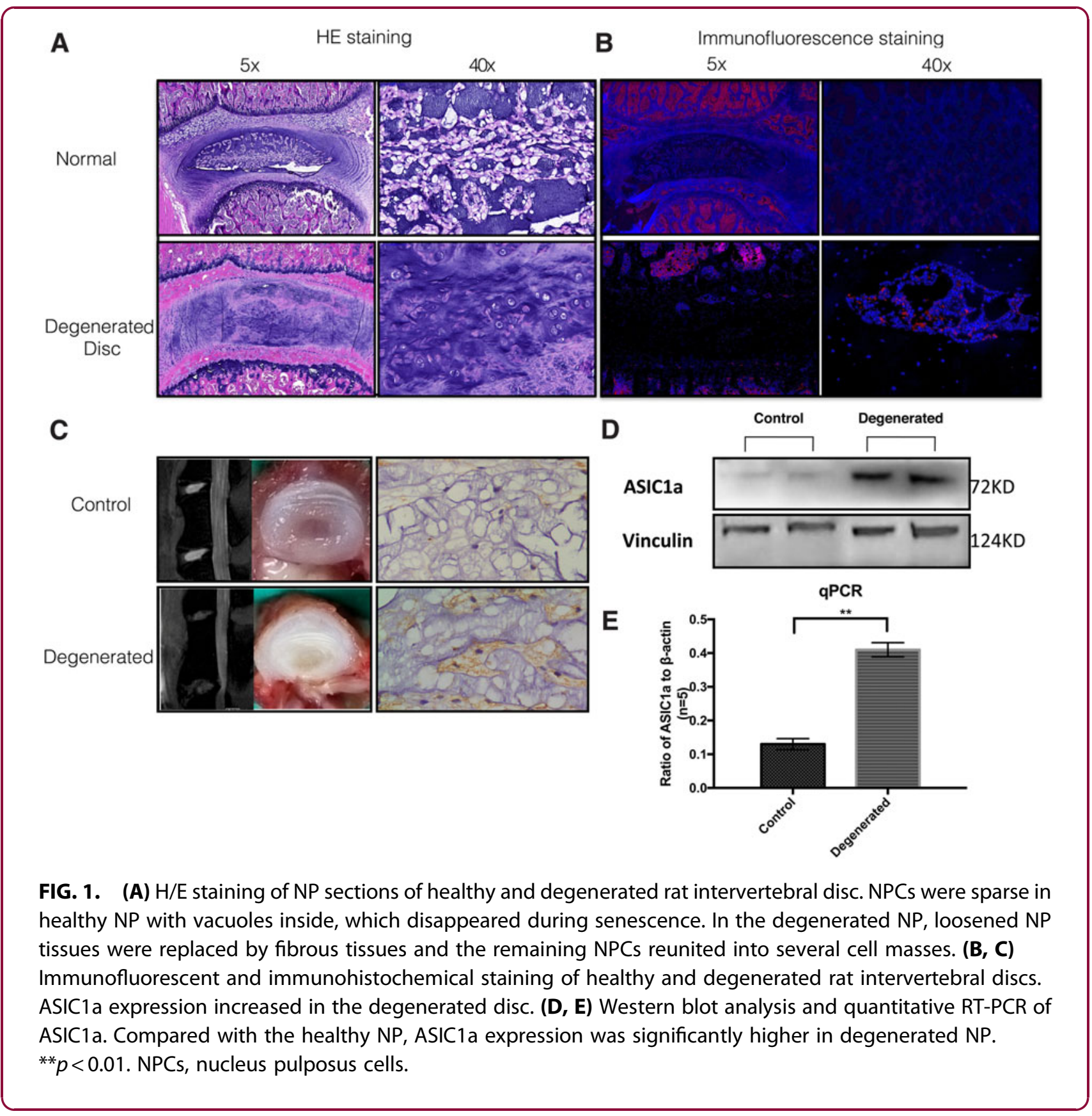

stimulus ( $\mathrm{pH}$ 6.5) containing $\mathrm{Ca}^{2+}$, and knockdown of ASICla inhibited acid-stimulated increase in $\left[\mathrm{Ca}^{2+}\right]_{\mathrm{i}}$ in rat NPCs (Fig. 2B, C).

The results of Annexin V-FITC/PI double-staining also showed that the percentage of apoptotic cells significantly increased at $\mathrm{pH} 6.5$ compared with that at $\mathrm{pH}$ 7.2. The fraction of apoptotic NPCs significantly decreased when ASICla was knocked down by siRNA (Fig. 2D, E).

\section{Acid-induced activation of ASIC1a}

regulated ER stress in NPCs

To examine the correlation between activation of ASICla and acid-induced ER stress in NPCs, Western blot analysis was performed to quantify the expression of ER stress markers. GRP78 was selected as the initiation of ER stress, XBP1 spliced/XBP1unspliced and phosphorylation of eIF2 $\alpha$ were taken as markers of unfolded protein response (UPR), and Caspase12 and 
A

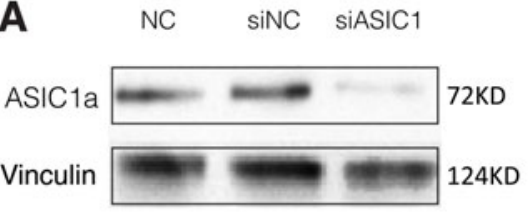

C

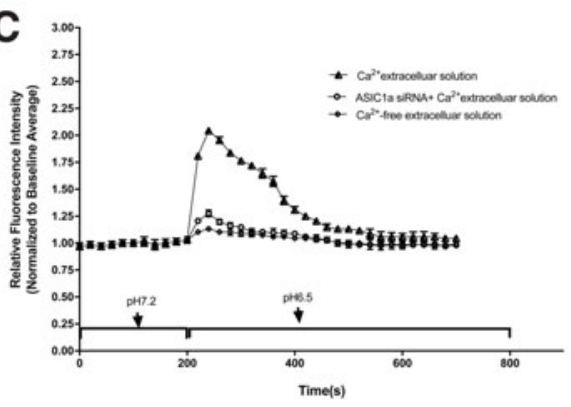

$\mathbf{E}$

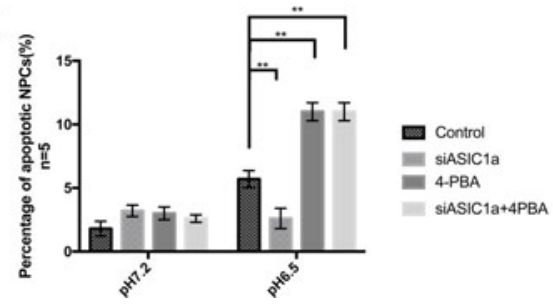

B

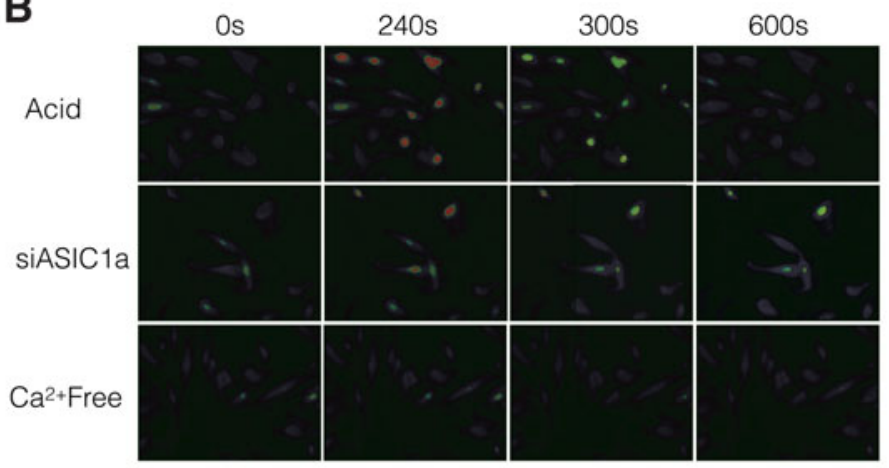

D

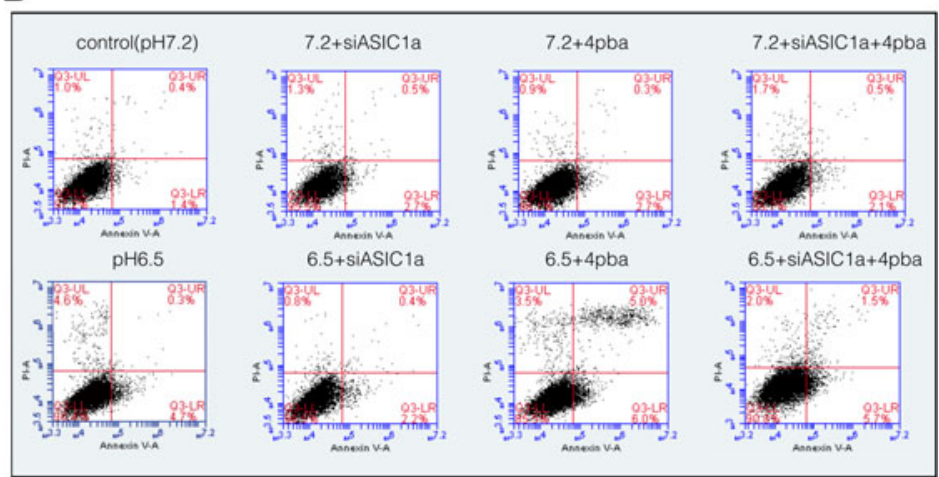

FIG. 2. (A) Compared with the two control groups, expression of ASIC1a was notably downregulated in the siASIC1a group. (B) $\mathrm{Ca}^{2+}$ imaging demonstrated that intracellular $\mathrm{Ca}^{2+}$ concentration significantly increased in response to the acidic extracellular solution (acid) containing $\mathrm{Ca}^{2+}$. (C) $\mathrm{Ca}^{2+}$ imaging indicated by Fura-2/AM at the indicated time. Acidic incubation (pH 6.5) failed to increase $\left[\mathrm{Ca}^{2+}\right]_{i}$ in the siASIC1a cells. (D, E) NPCs were incubated with acidity of pH 7.2 or 6.5 for $24 \mathrm{~h}$ (flow cytometry). Apoptotic cells = early apoptotic cells (lower right)+late apoptotic cells (upper right). Acid exposure induced apoptosis of rat NPCs. The ER stress inhibitor 4-PBA significantly promoted the apoptosis rate of NPCs under acid stimulus. ASIC1a knockdown reduced acidinduced apoptosis of NPCs, even in the presence of 4-PBA. ${ }^{* *} p<0.01$. ER, endoplasmic reticulum. 4-PBA, 4-phenylbutyrate.

CHOP were chosen as makers of ER stress-specific apoptosis. This analysis revealed that ER stress markers were upregulated along with acid-induced activation of ASIC1a, and blocking ASIC1a decreased the expression of ER stress markers, especially the proapoptotic CHOP and caspase12 (Fig. 3). The UPR-related marker XBP1 was not affected.

\section{Discussion}

The acidity in the NP environment is always a vital factor both in IVDD and biological regeneration. The $\mathrm{pH}$ of healthy NP ranges between 7.2 and 7.0. ${ }^{14}$ In a mildly degenerative state, the matrix acidity can drop to $\mathrm{pH}$ values of 6.8 to 6.5 . In the severe stage, the $\mathrm{pH}$ can reach 6.0 and even 5.7. Such $\mathrm{pH}$ values are suitable for the activation of ASICs. ${ }^{15-17}$

Cellular membranes have various $\mathrm{Ca}^{2+}$-transport and $\mathrm{Ca}^{2+}$-binding molecules that participate in $\mathrm{Ca}^{2+}$ movement into and out of cells and cellular organelles. Recent studies demonstrated a significant increase in ASIC1 and ASIC4-positive cells in the annulus fibrosus of degenerated IVD and marked upregulation of ASIC1, ASIC2, and ASIC3 in the nucleus pulposus. ${ }^{18}$ In addition, it also demonstrated that acid-induced $\left[\mathrm{Ca}^{+}\right]_{\mathrm{i}}$ elevation through ASIC1a is involved in endplate chondrocyte apoptosis. ${ }^{19}$ To the best of our knowledge, this is the first study to connect ASIC1a with ER stress. 


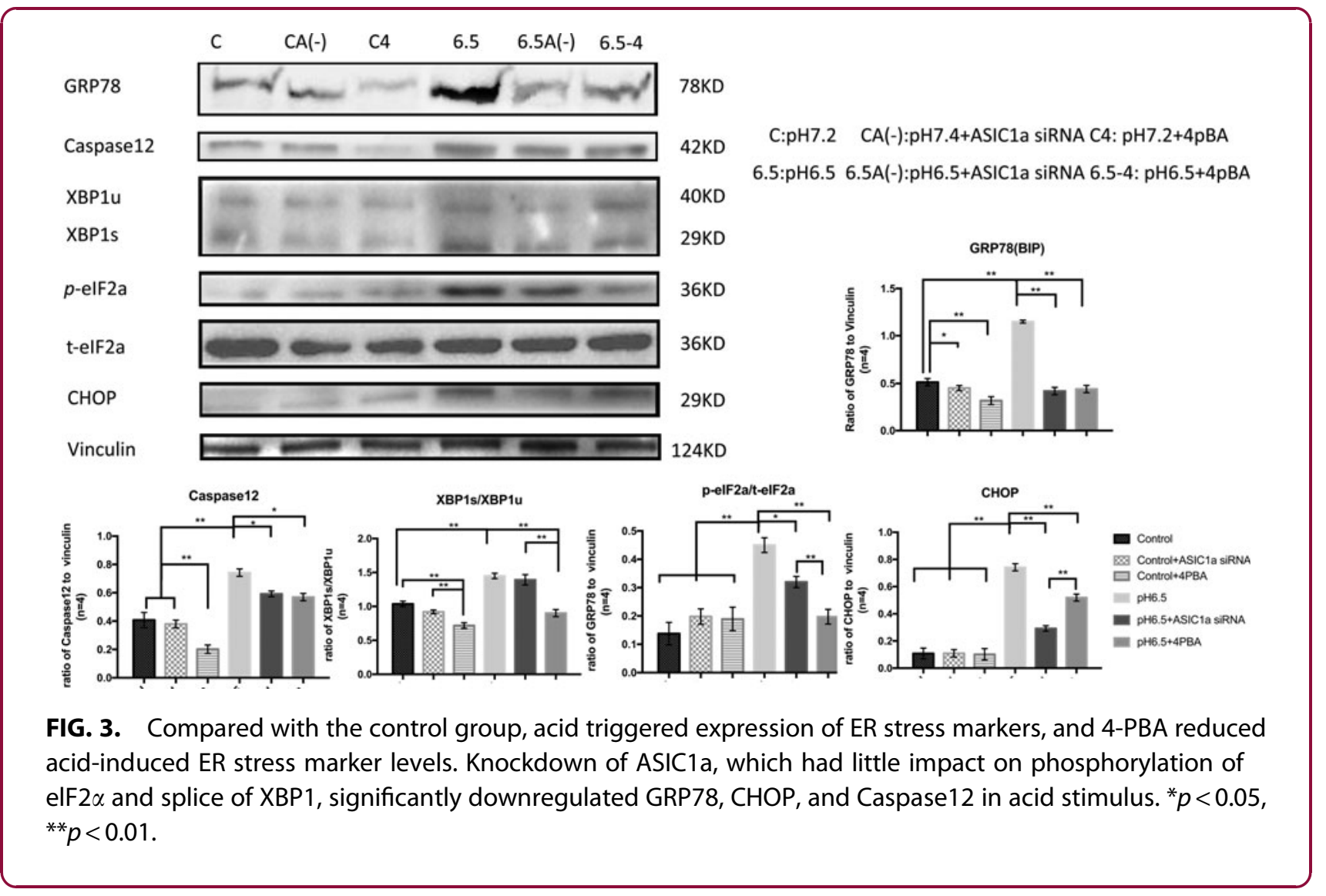

In this study, we proved the expression of ASICla in healthy and degenerated rat NPCs and investigated the role that ASICla plays in acidity-mediated $\mathrm{Ca}^{2+}$ influx. The results showed significantly higher ASICla expression in degenerated rat discs, at both the RNA and protein levels. We also examined the function of ASICla in degenerated rat NPCs. $\mathrm{Ca}^{2+}$ influx was significantly reduced in ASICla knockdown cells and in $\mathrm{Ca}^{2+}$-free extracellular solution, suggesting that ASICla in the cell membrane functions as a $\mathrm{Ca}^{2+}$ influx channel in rat NPCs. We previously discovered that even a transient $\mathrm{Ca}^{2+}$ signal overload activates a cascade of cytotoxic responses, resulting in the long-term activation of mitochondrial metabolism, and contributing to cellular apoptosis. ${ }^{5}$ An important and well-known aspect of cellular $\mathrm{Ca}^{2+}$ signaling is maintaining a balance between free versus bound $\mathrm{Ca}^{2+}$ in all cellular compartments. An imbalance in cellular calcium results in $\mathrm{Ca}^{2+}$ transport between ER-mitochondria contact sites, causing cellular stress. Such disruption of $\mathrm{Ca}^{2+}$ homeostasis in the ER leads to activation of ER stress responses, one of which is the UPR (Fig. 4).

A complex stress response program, such as that in ER stress, always acts in both directions in cellular stress, acting as a prosurvival response in mild stages and as a proapoptotic response in the prolonged stage. In a previous study, we revealed that ER stress was triggered in NP cell degeneration, primarily playing an antiapoptotic role in acid-induced NP cell apoptosis. Nevertheless, the mechanism of ER stress and transformation has been unclear. In the present study, we found that ASICla-activated $\mathrm{Ca}^{2+}$ influx is one of the initiation factors of ER stress and is associated with proapoptotic ER stress pathways. We chose to use 4-PBA (a classical blocker of ER stress) in our experiments. While 4-PBA disturbs the recognition of unfolded proteins by molecular chaperon, it decreases the detachment of GRP78 and the downstream UPR pathway. Compared with 4-PBA intervention, blockade of the ASICla pathway significantly reduced the level of GRP78, a specific marker of ER stress activation. The multifunctional nature of the ER enables the ER membrane to modulate its own luminal $\mathrm{Ca}^{2+} \mathrm{dy}$ namics and generate appropriate signals to maintain balanced homeostasis. ${ }^{20}$ GRP78 (or BiP) is an especially important $\mathrm{Ca}^{2+}$-binding protein, and $\mathrm{Ca}^{2+}$ buffer is involved in sensing accumulation of misfolded 


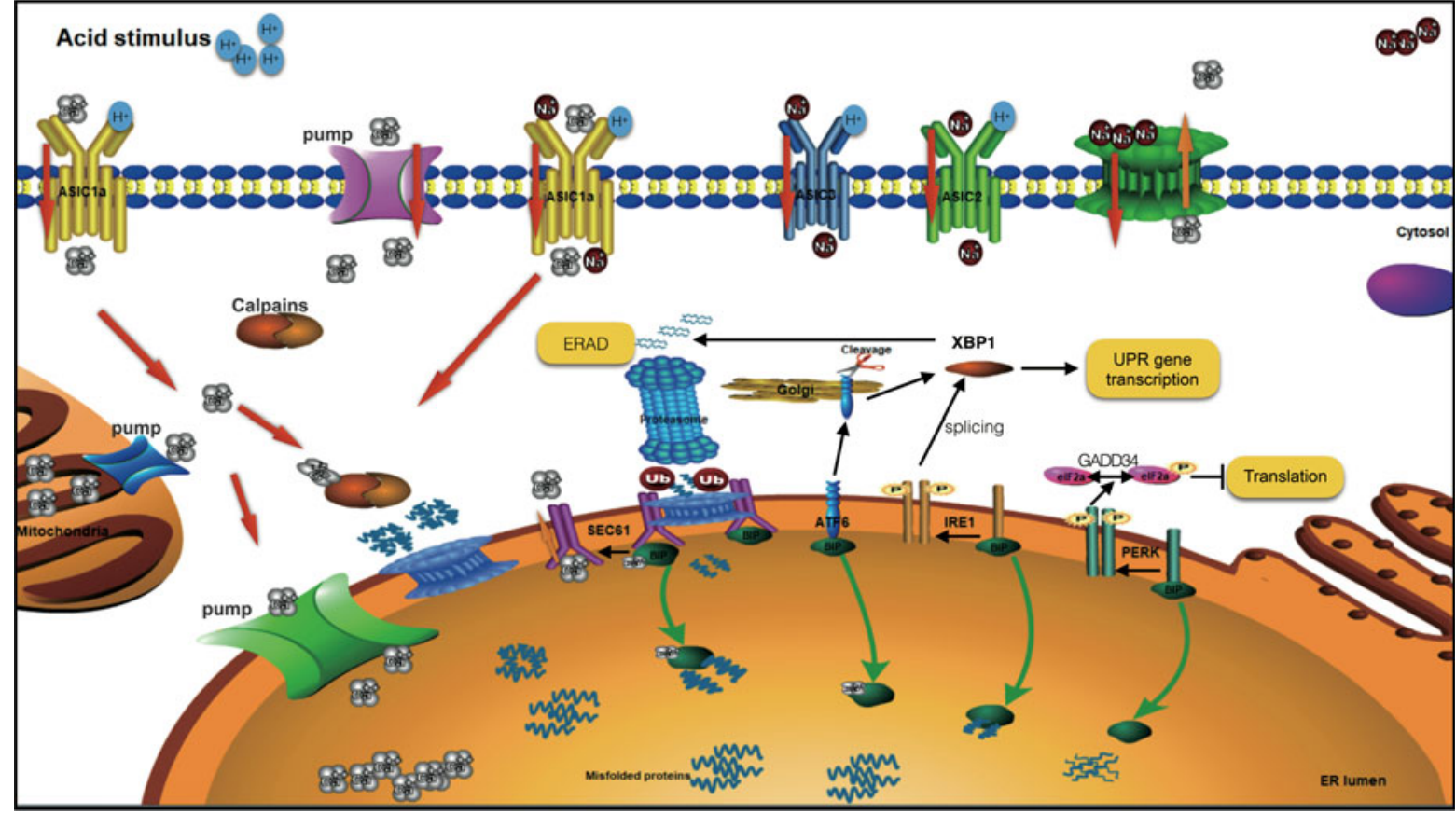

FIG. 4. Schematic diagram highlighting ASIC1a and UPR signaling. ASIC, acid-sensing ion channel; ATF6, activating transcription factor 6; BiP, binding immunoglobulin protein; ERAD, ER-associated degradation; GRP78, glucose-regulated protein 78; IRE, inositol-requiring enzyme; PERK, protein kinase-like ER kinase; UPR, unfolded protein response.

proteins in the ER. In addition, GRP78 may act as a joint node of ASICla and acid-induced ER stress.

CHOP and Caspase12, which are key proteins in ER stress-associated apoptosis, were also considerably downregulated, whereas the UPR-related ratio of XBP1s/ $\mathrm{XBP} 1 \mathrm{u}$ and the phosphorylation of eIF $2 \alpha$ barely decreased. These findings suggest that acid-induced activation of $\mathrm{Ca}^{2+}$-permeable ASICla predominantly affects the proapoptotic ER stress pathway and that the UPR maintains the homeostasis of the internal environment. Flow cytometry also demonstrated that knockdown of AISC1a markedly reduces acid-induced NP cell apoptosis, and also suggests that ASICla would be a good target to reduce NPCs apoptosis in an acidic environment.

The present study has several limitations. First, as a preliminary study, we only demonstrated that the activation of ASIC1a is involved in rat NPCs apoptosis and it may play a role in acidosis-mediated ER stress. Although this study provides a novel insight into IVD biology, additional research is necessary to strengthen this finding. Second, ASICla is not the only $\mathrm{Ca}^{2+}$ channel in the cell membrane that is acti- vated in acidic environments, and intracellular calcium is not the only initiator of ER stress. In our experiments, we also found that the expression of GRP78 was markedly upregulated when we incubated siASIC1a-NPCs with 4-PBA in acidic environments, suggesting that some other cellular pathways had been activated to initiate ER stress. Third, we examined ER stress from a functional perspective with some representative markers. The temporal sequence of ER stress activation needs to be further examined to better understand ER stress and calcium homeostasis. Fourth, besides ASIC1a, ASIC3 was also found to be upregulated in acid-induced cell stress in our study. As reported, ${ }^{15,16,21}$ ASIC3 may be a cofactor in acid stimulation and calcium influx, and it has also been described as being involved in the expression of proinflammatory cytokines, ${ }^{17}$ but further studies are required to substantiate this idea.

\section{Conclusion}

Findings of this study support an essential role for ASIC1a in acid-induced NPCs death. Activation of ASICla and 
the ASICla-mediated ER stress pathway primarily participates in NPCs apoptosis. ASICla may also be a prospective target for treating NP degeneration.

\section{Acknowledgments}

The research was supported by grants from the National Natural Science Foundation of China (81572170, $81572190,81702203,81702190$, and 81702201), the Fundamental Research Funds for the Central Universities and Postgraduate Research \& Practice Innovation Program of Jiangsu Province (KYLX16_0302, KYCX17_0182, 2242016K40149, and 2242017K3DN06), and the Research Funds of the Jiangsu Provincial Commission of Health and Family Planning (H201533).

\section{Author Disclosure Statement}

No competing financial interests exist.

\section{Reference}

1. Vos $T$, Flaxman AD, Naghavi $M$, et al. Years lived with disability (YLDs) for 1160 sequelae of 289 diseases and injuries 1990-2010: a systematic analysis for the Global Burden of Disease Study 2010. Lancet. 2012;380: 2163-2196.

2. Luo X, Pietrobon R, Sun SX, et al. Estimates and patterns of direct health care expenditures among individuals with back pain in the United States. Spine (Phila Pa 1976). 2004;29:79-86.

3. Freemont AJ, Peacock TE, Goupille $P$, et al. Nerve ingrowth into diseased intervertebral disc in chronic back pain. Lancet. 1997;350:178-181.

4. Sakai D, Nakamura Y, Nakai T, et al. Exhaustion of nucleus pulposus progenitor cells with ageing and degeneration of the intervertebral disc. Nat Commun. 2012;3:1264.

5. Cai $F$, Wang F, Hong $X$, et al. Acid-sensing ion channel 1a regulates the survival of nucleus pulposus cells in the acidic environment of degenerated intervertebral discs. Iran J Basic Med Sci. 2016;19:812-820.

6. Xie ZY, Chen L, Wang F, et al. Endoplasmic reticulum stress is involved in nucleus pulposus degeneration and attenuates low $\mathrm{pH}$-induced apoptosis of rat nucleus pulposus cells. DNA Cell Biol. 2017;36:627-637.

7. Sharma V, Kaur R, Bhatnagar A, et al. Low-pH-induced apoptosis: role of endoplasmic reticulum stress-induced calcium permeability and mitochondria-dependent signaling. Cell Stress Chaperones. 2015;20:431440.

8. Mesitov MV, Soldatov RA, Zaichenko DM, et al. Differential processing of small RNAs during endoplasmic reticulum stress. Sci Rep. 2017;7:46080.

9. Wemmie JA, Taugher RJ, Kreple CJ. Acid-sensing ion channels in pain and disease. Nat Rev Neurosci. 2013;14:461-471.

10. Benarroch EE. Acid-sensing cation channels: structure, function, and pathophysiologic implications. Neurology. 2014;82:628-635.

11. Wang F, Cai F, Shi R, et al. Hypoxia regulates sumoylation pathways in intervertebral disc cells: implications for hypoxic adaptations. Osteoarthritis Cartilage. 2016;24:1113-1124.

12. Wang F, Cai F, Shi R, et al. Aging and age related stresses: a senescence mechanism of intervertebral disc degeneration. Osteoarthritis Cartilage. 2016;24:398-408.

13. Cai F, Zhu L, Wang F, et al. The paracrine effect of degenerated disc cells on healthy human nucleus pulposus cells is mediated by MAPK and NFkappaB pathways and can be reduced by TGF-beta1. DNA Cell Biol. 2017; 36:143-158.

14. Kitano T, Zerwekh JE, Usui Y, et al. Biochemical changes associated with the symptomatic human intervertebral disk. Clin Orthop Relat Res. 1993: 372-377.

15. Uchiyama Y, Cheng CC, Danielson KG, et al. Expression of acid-sensing ion channel 3 (ASIC3) in nucleus pulposus cells of the intervertebral disc is regulated by p75NTR and ERK signaling. J Bone Miner Res. 2007;22:19962006.
16. Silagi ES, Schoepflin ZR, Seifert EL, et al. Bicarbonate recycling by HIF-1dependent carbonic anhydrase isoforms 9 and 12 is critical in maintaining intracellular $\mathrm{pH}$ and viability of nucleus pulposus cells. J Bone Miner Res. 2017. [Epub ahead of print]; DOI:10.1002/jbmr.3293.

17. Gilbert HT, Hodson N, Baird $\mathrm{P}$, et al. Acidic $\mathrm{pH}$ promotes intervertebral disc degeneration: acid-sensing ion channel -3 as a potential therapeutic target. Sci Rep. 2016;6:37360.

18. Cuesta A, Del Valle ME, Garcia-Suarez O, et al. Acid-sensing ion channels in healthy and degenerated human intervertebral disc. Connect Tissue Res. 2014;55:197-204.

19. Li X, Wu FR, Xu RS, et al. Acid-sensing ion channel 1a-mediated calcium influx regulates apoptosis of endplate chondrocytes in intervertebral discs. Expert Opin Ther Targets. 2014;18:1-14.

20. Krebs J, Agellon LB, Michalak M. $\mathrm{Ca}(2+)$ homeostasis and endoplasmic reticulum (ER) stress: an integrated view of calcium signaling. Biochem Biophys Res Commun. 2015;460:114-121.

21. Zhu S, Zhou HY, Deng SC, et al. ASIC1 and ASIC3 contribute to acidityinduced EMT of pancreatic cancer through activating $\mathrm{Ca2}+/$ RhoA pathway[J]. Cell Death Dis. 2017;8:e2806.

Cite this article as: Xie Z-Y, Chen L, Zhang C, Liu L, Wang F, Cai F, Wang X-H, Shi R, Sinkemani A, Yu H-M, Hong X, Wu X-T (2018) Acidsensing ion channel 1 a regulates fate of rat nucleus pulposus cells in acid-stimulus via endoplasmic reticulum stress, BioResearch Open Access 7:1, 2-9, DOI: 10.1089/biores.2017.0049.

$\begin{aligned} & \text { Abbreviations Used } \\ & 4-\mathrm{PBA}= \text { 4-phenylbutyrate } \\ & \mathrm{ASIC}=\text { acid-sensing ion channels } \\ & \mathrm{ATF} 6=\text { activating transcription factor } 6 \\ & \mathrm{BiP}=\text { binding immunoglobulin protein } \\ & \mathrm{BSA}=\text { bovine serum albumin } \\ & \mathrm{DAPI}=4 \text {,6-diamidino-2-phenylindole } \\ & \mathrm{ER}=\text { endoplasmic reticulum } \\ & \mathrm{ERAD}=\text { ER-associated degradation } \\ & \mathrm{FITC}=\text { fluorescein isothiocyanate } \\ & \mathrm{GRP78}=\text { glucose-regulated protein } 78 \\ & \mathrm{IRE}=\text { inositol-requiring enzyme } \\ & \mathrm{IVDD}=\text { human intervertebral disc degeneration } \\ & \mathrm{MRI}=\text { magnetic resonance imaging } \\ & \mathrm{NC}=\text { negative control } \\ & \mathrm{NPCS}=\text { nucleus pulposus cells } \\ & \mathrm{PERK}=\text { protein kinase-like ER kinase } \\ & \mathrm{PI}=\text { propidium iodide } \\ & \mathrm{RT}-\mathrm{PCR}=\text { real-time-polymerase chain reaction } \\ & \mathrm{UPR}=\text { unfolded protein response }\end{aligned}$

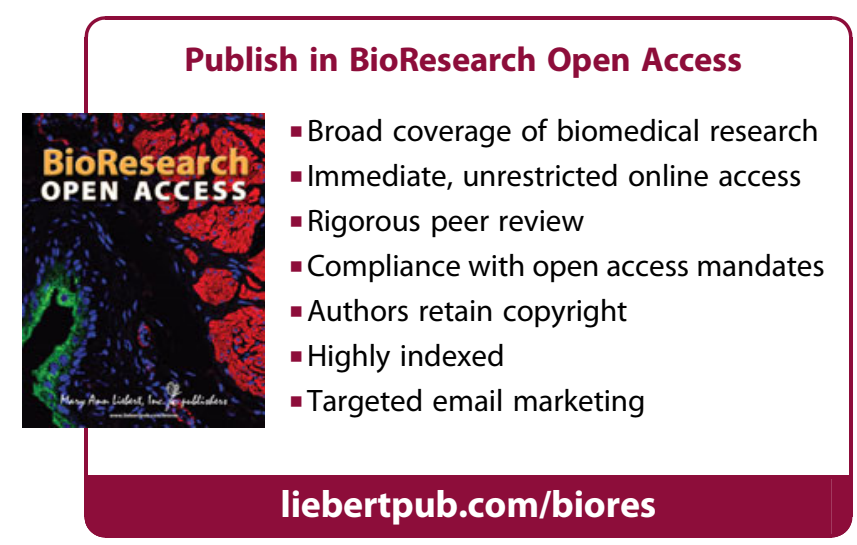

